\title{
TOPOLOGICAL RATIONALIZATION OF A CLASS OF MEROMORPHIC FUNCTIONS
}

\author{
A. MEZIANI
}

(Communicated by Clifford J. Earle, Jr.)

\begin{abstract}
We prove that a class of germs at $0 \in \mathbf{C}^{2}$ of meromorphic functions can be transformed, via homeomorphisms, into rational functions.
\end{abstract}

\section{INTRODUCTION}

We prove in this note that if $M(x, y)$ is the germ at $0 \in \mathbf{C}^{2}$ of a pure meromorphic function whose blow-up $\widetilde{M}=M \circ E$, where $E$ is the blow-up of $\mathbf{C}^{2}$ at 0 , has no essential singularities on $E^{-1}(0)$, then $M$ is topologically conjugate to a rational function $R(x, y)$. That is, there is a homeomorphism $\Phi$ of a neighborhood of $0 \in \mathrm{C}^{2}$ such that $M \circ \Phi=R$.

D. Cerveau and J. F. Mattei have studied [1] finite determination for multiform functions. In particular, they prove rationalization for germs of meromorphic functions with critical set reduced to $\{0\}$. They also show that germs at $0 \in \mathbf{C}^{2}$ of holomorphic functions are conjugate to polynomials.

We begin this note by recalling briefly the blow-up of $\mathbf{C}^{2}$ at 0 , and then we define the class of meromorphic functions that we will be dealing with. Next, we prove an intermediate result: a particular conjugation of germs of analytic varieties. Finally, we state and prove the main result.

\section{Definitions}

In this section, we recall very briefly the blow-up of $\mathbf{C}^{2}$ at 0 (see [4]) and define a class of meromorphic functions, called here class A. Some examples will follow.

The blow-up of $\mathbf{C}^{2}$ at 0 is the subset of $\mathbf{C}^{2} \times \mathbf{C P} \mathbf{P}^{1}$, where $\mathbf{C} \mathbf{P}^{1}$ is the complex projective space of dimension 1 , given as

$$
\mathbf{C}_{0}^{2}=\left\{(p,[p]) \in\left(\mathbf{C}^{2}-\{0\}\right) \times \mathbf{C} \mathbf{P}^{1}\right\} \cup\left(\{0\} \times \mathbf{C} \mathbf{P}^{1}\right),
$$

where [ ]: $\mathbf{C}^{2}-\{0\} \mapsto \mathbf{C} \mathbf{P}^{1}$ is the quotient map. It is shown, in [4] for example, that $\mathbf{C}_{0}^{2}$ is a two-dimensional complex manifold. Moreover, $\mathbf{C}_{0}^{2}$ is covered by

Received by the editors April 3, 1989.

1980 Mathematics Subject Classification (1985 Revision). Primary 32A20. 
two charts $(x, t),(s, y) \quad\left(t, s\right.$ are the canonical coordinates of $\left.\mathbf{C P}^{1}\right)$ related by

$$
s=\frac{1}{t} \text { and } y=x t .
$$

The first projection $E: \mathbf{C}_{0}^{2} \mapsto \mathbf{C}^{2}$, called the blow-up map, is given in the charts $(x, t),(s, y)$ by

$$
E(x, t)=(x, x t) \text { and } E(s, y)=(s y, y) .
$$

$E$ is a biholomorphism from $\mathbf{C}_{0}^{2}-E^{-1}(0)$ onto $\mathbf{C}^{2}-\{0\}$. The exceptional divisor $E^{-1}(0)$ is identified with $\mathbf{C} \mathbf{P}^{1}$.

A meromorphic function $M$ on a two-dimensional complex manifold $\mathscr{M}$ is said to have an essential singularity at $p_{0} \in \mathscr{M}$ if, near $p_{0}$, the function $M$ is expressed as a quotient $\alpha / \beta$, with $\alpha, \beta$ germs at $p_{0}$ of coprime holomorphic functions and $\alpha\left(p_{0}\right)=\beta\left(p_{0}\right)=0$. Equivalently, $M$ has an essential singularity at $p_{0}$ if, in a neighborhood of $p_{0}$, all the level sets of $M$ pass through $p_{0}$. In this case, as in [1], we say that $M$ is a pure meromorphic function at $p_{0}$.

Definition 1.1. A germ at $0 \in \mathbf{C}^{2}$ of a pure meromorphic function $M(x, y)$ is said to be in the class $A$ if the map $\widetilde{M}=M \circ E: \mathbf{C}_{0}^{2} \mapsto \overline{\mathbf{C}}$, defined in a neighborhood of $E^{-1}(0)=\mathbf{C P}^{1} \subset \mathbf{C}_{0}^{2}$, has no essential singularities.

Remark 1.1. It is easy to see, as a consequence of the desingularization of foliations defined by level sets of meromorphic functions (see [3] for example), that if $M$ is the germ at $0 \in \mathbf{C}^{2}$ of a pure meromorphic function, then there is a proper holomorphic map $\Pi: \mathscr{M} \mapsto \mathbf{C}^{2}$, obtained by composition of blow-ups, such that the lift $M \circ \Pi$ of $M$ to $\mathscr{M}$ has no essential singularities. Hence, the class $A$ is the set of all meromorphic functions for which $\mathscr{M}=\mathrm{C}_{0}^{2}$.

Example 1. The function

$$
M(x, y)=\frac{x^{3}+x y^{2}+\alpha(x, y)}{y^{3}+\beta(x, y)},
$$

where $\alpha, \beta$ are holomorphic functions of order $\geq 4$, is in the class $A$. The blow-up of $M$ is given in the charts $(x, t),(s, y)$ respectively by

$$
\widetilde{M}(x, t)=\frac{1+t^{2}+\left(\alpha(x, t x) / x^{3}\right)}{y^{3}+\left(\beta(x, t x) / x^{3}\right)}, \quad \widetilde{M}(s, y)=\frac{s^{3}+s+\left(\alpha(s y, y) / y^{3}\right)}{1+\left(\beta(s y, y) / y^{3}\right)} .
$$

So $\widetilde{M}$ has no essential singularities on $\mathbf{C P}^{1}=\{x=0\} \cup\{y=0\}$.

Example 2. The meromorphic function $M(x, y)=\left(y^{2}+x^{3}\right) / x y$ is not in the class $A$. Its blow-up $\widetilde{M}(x, t)=\left(t^{2}+x\right) / t$ has an essential singularity at $(x=0, t=0)$. A blow-up of $\mathbf{C}_{0}^{2}$ at $(x=0, t=0)$ will produce a meromorphic function with no essential singularities. 
Remark 1.2. The functions of the form $x^{r} / y^{s}$, where $r, s$ are positive integers and $r>s$, are not in the class $A$. However, we characterized in [3] all meromorphic functions that are conjugate to the rational functions of the form $R\left(x^{r} / y^{s}\right)$, where $R$ is a rational function on $\overline{\mathbf{C}}$.

The following proposition characterizes the class $A$.

Proposition 1.1. Let $M(x, y)=\alpha(x, y) / \beta(x, y)$ be the germ at $0 \in \mathbf{C}^{2}$ of a pure meromorphic function and let

$$
\alpha(x, y)=\sum_{i \geq n} P_{i}(x, y) \quad \text { and } \beta(x, y)=\sum_{j \geq m} Q_{j}(x, y)
$$

be the Taylor expansions of $\alpha$ and $\beta\left(P_{i}\right.$ and $Q_{j}$ are homogeneous polynomials with respective degrees $i$ and $j$ ). Then $M$ is in the class $A$ if and only if $n=m$ and $P_{n}, Q_{n}$ are coprime.

Proof. We first show that if $n=m$ and $P_{n}, Q_{n}$ are coprime, then $M$ is in the class $A$. The blow-up $\widetilde{M}=M \circ E$ of $M$ is given in the charts $(x, t),(s, y)$ by

$$
\begin{aligned}
& \widetilde{M}(x, t)=\frac{P_{n}(1, t)+x P_{n+1}(1, t)+\ldots}{Q_{n}(1, t)+x Q_{n+1}(1, t)+\ldots}, \\
& \widetilde{M}(s, y)=\frac{P_{n}(s, 1)+y P_{n+1}(s, 1)+\ldots}{Q_{n}(s, 1)+y Q_{n+1}(s, 1)+\ldots},
\end{aligned}
$$

Since $P_{n}(1, t)$ and $Q_{n}(1, t)$ (resp. $P_{n}(s, 1)$ and $\left.Q_{n}(s, 1)\right)$ have no common root, then $\widetilde{M}$ has no essential singularities on $E^{-1}(0)$.

Conversely, let us show that if $M$ is in the class $A$, then $n=m$ and $P_{n}$, $Q_{n}$ are coprime. By contradiction, first if $n=m+k>m$, then

$$
\begin{aligned}
& \widetilde{M}(x, t)=\frac{x^{k}\left(P_{m+k}(1, t)+x P_{m+k+1}(1, t)+\ldots\right)}{Q_{m}(1, t)+x Q_{m+1}(1, t)+\ldots}, \\
& \widetilde{M}(s, y)=\frac{y^{k}\left(P_{m+k}(s, 1)+y P_{m+k+1}(s, 1)+\ldots\right)}{Q_{m}(s, 1)+y Q_{m+1}(s, 1)+\ldots} .
\end{aligned}
$$

So, if $t_{0}$ (resp. $s_{0}$ ) is a root of $Q_{m}(1, t)$ (resp. $\left.Q_{m}(s, 1)\right)$, then the point $\left(x=0, t=t_{0}\right) \quad\left(\right.$ resp. $\left.\left(s=s_{0}, y=0\right)\right)$ would be an essential singularity of $\widetilde{M}$. The second eventuality, $n=m$ and $P_{n}, Q_{n}$ are not coprime, also cannot occur. Indeed, $P_{n}(1, t)$ and $Q_{n}(1, t)$ would have a common root $t_{0}$, and then

$$
\begin{aligned}
\widetilde{M}(x, t) & =\frac{P_{n}(1, t)+x P_{n+1}(1, t)+\ldots}{Q_{n}(1, t)+x Q_{n+1}(1, t)+\ldots} \\
& =\frac{\left(t-t_{0}\right) P_{n}^{*}(1, t)+x P_{n+1}(1, t)+\ldots}{\left(t-t_{0}\right) Q_{n}^{*}(1, t)+x Q_{n+1}(1, t)+\ldots}
\end{aligned}
$$

would have an essential singularity at $\left(x=0, t=t_{0}\right)$. 


\section{AN INTERMEDIATE RESULT}

We prove in this section the existence of a particular homeomorphism between two germs of analytic varieties that coincide to high order at $0 \in \mathbf{C}^{2}$. We will denote by $\mathbf{C}_{c}$ the complex line in $\mathbf{C}^{2}$ given by $y=c x$, where $c$ is a complex number.

Proposition 2.1. Let $f(x, y)$ be the germ at $0 \in \mathbf{C}^{2}$ of a holomorphic function and let $f_{k}(x, y)$ be its $k$ th jet. Define

$$
V(f)=\{(x, y) ; f(x, y)=0\} \text { and } V\left(f_{k}\right)=\left\{(x, y) ; f_{k}(x, y)=0\right\} .
$$

Then, for $k$ large enough, there is a germ of a homeomorphism $\Phi$ at $0 \in \mathbf{C}^{2}$ such that

$$
\Phi(V(f))=V\left(f_{k}\right) \text { and } \Phi\left(\mathbf{C}_{c}\right)=\mathbf{C}_{c}
$$

for every $c \in \mathbf{C}$.

The proof of this proposition is based upon some lemmas.

Lemma 2.1. Consider a family $D\left(p_{i}, r_{i}\right), i=1, \ldots, N$, of $N$ disjoint discs in the plane $\mathbf{R}^{2}$, with respective centers $p_{i}$ and radii $r_{i}$. For every $i$, let $q_{i}$ be a point in $D\left(p_{i}, r_{i}\right)$. Then there is a homeomorphism $\phi: \mathbf{R}^{2} \mapsto \mathbf{R}^{2}$ such that:

$$
\begin{gathered}
\phi(p)=p \quad \text { if } p \notin \bigcup_{i=1}^{N} D\left(p_{i}, r_{i}\right) \\
\phi\left(p_{i}\right)=q_{i} \text { for } i=1, \ldots, N .
\end{gathered}
$$

We leave the elementary proof of this result to the reader.

Lemma 2.2. Let $f(x, y)$ be the germ at $0 \in \mathbf{C}^{2}$ of an irreducible holomorphic function of order $n$. Then, we can assume, after a linear change of coordinates, that

$$
f(x, y)=y^{n}+P_{n+1}(x, y)+\ldots+P_{n+k}(x, y)+\ldots,
$$

where the $P_{n+j}$ 's are homogeneous polynomials of degree $n+j$. Furthermore, if $n>1$, then there is a $k$ such that $P_{n+k}(x, 0)$ is not identically zero.

Proof. The Taylor expansion of $f$ is $f(x, y)=\sum_{j \geq n} P_{j}(x, y)$, where the $P_{j}$ 's are homogeneous polynomials of degree $j$. Recall from [4] that the irreducibility of $f$ implies that the tangent cone of the variety $\{(x, y) ; f(x, y)=0\}$ is reduced to a point. That is, the set of points on $\mathbf{C P}^{1}$ on which $P_{n}(1, t)$ or $P_{n}(s, 1)=0$ is reduced to one point. We can assume, after a linear change of coordinates if necessary, that $s=0$ is not a root of $P_{n}(s, 1)$. So, the irreducibility of $f$ implies that $P_{n}(1, t)=\left(t-t_{0}\right)^{i}$ for some $t_{0} \in \mathbf{C}$ and $i \leq n$. By a linear change of coordinates, we can assume $t_{0}=0$. Now, if $n>i$, then $P_{n}(s, 1)=s^{n-i}$ would have a root at $s=0$, and this is impossible. So $i=n$ and $P_{n}(x, y)=y^{n}$. Finally, if $n>1$ and $P_{n+j}(x, 0)=0$ for every 
$j \geq 1$, then $y$ would be a factor of $f$ and $f$ would be reducible. The lemma is proved.

Lemma 2.3. Let $f(x, y)=y^{n}+P_{n+1}(x, y)+\ldots+P_{n+k}(x, y)+\ldots$, be an irreducible holomorphic function and $k$ be the first integer such that $P_{n+k}(x, 0) \neq 0$. Then there is a positive constant $K$ such that, for every $c \in \mathbf{C}, 0<|c|<K$, the variety $V(f)=\{f=0\}$ intersects the line $\mathbf{C}_{c}$ at 0 and $k$ other points $p^{1}(c), \ldots, p^{k}(c)$ which vary continuously with $c$ and $p^{1}(0)=\ldots=p^{k}(0)=0$.

Proof. Let $c$ be fixed in $\mathbf{C}$. Then

$$
V(f) \cap \mathbf{C}_{c}=\left\{(x, c x) \in \mathbf{C}^{2} ; f(x, c x)=0\right\},
$$

i.e., $V(f) \cap \mathbf{C}_{c}$ is given by the equation

$$
x^{n}\left(c^{n}+P_{n+1}(1, c) x+\ldots+P_{n+k}(1, c) x^{k}+\ldots\right)=0 .
$$

Since $P_{n+j}(1,0)=0$ if $j<k$ and $P_{n+k}(1,0) \neq 0$, then similar arguments as that used in the proof of Theorem 4.13 of Chapter 2 in [2] and the irreducibility of $f$ show that the equation

$$
c^{n}+P_{n+1}(1, c) x+\ldots+P_{n+k}(1, c) x^{k}+\ldots=0
$$

has $k$ distinct and continuous roots $p^{1}(c), \ldots, p^{k}(c)$, provided that $c \neq 0$ is sufficiently small and $p^{1}(0)=\ldots=p^{k}(0)=0$.

Proof of the proposition. We start by proving the proposition in the case where the tangent cone of $V(f)$ is reduced to one point. Let $K>0$ be such that, for every $c \in \mathbf{C}, 0<|c|<K$, we have

$$
V(f) \cap \mathbf{C}_{c}=\left\{0, p^{1}(c), \ldots, p^{k}(c)\right\}
$$

(see Lemma 2.3). Consider the homotopy

$$
g_{t}=f+t\left(f_{l}-f\right), \quad 0 \leq t \leq 1,
$$

where $f_{l}$ is the $l$ th jet of $f$. Since $f$ and $g_{t}$ coincide to order $l$ at 0 , then, for $l$ sufficiently large, we also have

$$
V\left(g_{t}\right) \cap \mathbf{C}_{c}=\left\{0, p_{t}^{1}(c), \ldots, p_{t}^{k}(c)\right\},
$$

and the functions $p_{t}^{j}(c)$ 's are continuous in $c$ and $t$. So, for every $j$, we can find a continuous function $r_{j}(c), r_{j}(c)>0$ and $r_{j}(0)=0$ such that

$$
\left\{p_{t}^{j}(c), 0 \leq t \leq 1\right\} \subset D\left(p^{j}(c), r_{j}(c)\right)
$$

and

$$
D\left(p^{j}(c), r_{j}(c)\right) \cap D\left(p^{i}(c), r_{i}(c)\right)=\varnothing \quad \text { if } j \neq i
$$


Next, for $c$ fixed, $|c|<K$, consider, as in Lemma 2.1, a homeomorphism $\phi_{c}$ of the complex line $\mathbf{C}_{c}$ such that

$$
\begin{gathered}
\phi_{c}(p)=p \quad \text { if } p \in \mathbf{C}_{c}-\bigcup_{i=1}^{k} D\left(p^{i}(c), r_{i}(c)\right), \\
\phi_{c}\left(p^{i}(c)\right)=p_{1}^{i}(c) \quad \text { for } i=1, \ldots, k .
\end{gathered}
$$

Note that if $U$ is a small neighborhood of $0 \in \mathbf{C}^{2}$ and $c$ is such that $K / 2<$ $|c|<K$, then $\phi_{c}(p)=p$ if $p \in U \cap \mathbf{C}_{c}$. So, we can define a homeomorphism $\Phi:\left(\mathbf{C}^{2}, 0\right) \mapsto\left(\mathbf{C}^{2}, 0\right)$ by

$$
\begin{aligned}
& \Phi(p)=p \quad \text { if } p \notin\{(x, y) ; y=c x \text { and }|c|<K\} \\
& \Phi(p)=\phi_{c}(p) \quad \text { if } p \in \mathbf{C}_{c} \text { and }|c|<K .
\end{aligned}
$$

In this case $\Phi$ is the desired homeomorphism of the proposition.

In the general case, the tangent cone of $V(f)$ is not necessarily reduced to a point; we decompose

$$
V(f)=V^{1} \cup \ldots \cup V^{N},
$$

where each $V^{j}$ has a tangent cone reduced to a point $a_{j}$. Similarly, we decompose

$$
V\left(f_{k}\right)=V_{k}^{1} \cup \ldots \cup V_{k}^{N},
$$

where the tangent cone of $V_{k}^{j}$ is the point $a_{j}$. By reducing the neighborhood $U$ of $0 \in \mathbf{C}^{2}$, we can assume that, for every $j$, there is a $K_{j}$ such that $V^{j} \cap \mathbf{C}_{c}=$ $\{0\}$ if $\left|c-a_{j}\right| \geq K_{j}$ and the discs $D\left(a_{j}, K_{j}\right)$ are mutually disjoint. Now we can define a homeomorphism $\Phi$ as follows:

$$
\begin{aligned}
& \Phi(p)=p \quad \text { if } p \notin \bigcup_{j=1}^{N}\left\{(x, y) ; y=c x \text { and }\left|c-a_{j}\right| \geq K\right\} \\
& \Phi(p)=\Phi_{j}(p) \quad \text { if } p \in\left\{(x, y) ; y=c x \text { and }\left|c-a_{j}\right|<K_{j}\right\}
\end{aligned}
$$

where $\Phi_{j}$ is a homeomorphism, as in the previous case, corresponding to $V^{j}$. The proposition is therefore proved.

\section{Statement AND PROOF OF THE MAIN RESUlt}

Theorem. Let $M(x, y)$ be the germ at $0 \in \mathbf{C}^{2}$ of a meromorphic function in the class $A$. Then $M$ is topologically conjugate to a rational function.

The proof of this result needs two lemmas. First, we adopt the following notation: if $V(f)$ is the germ of the variety $\{f=0\}$, we will denote by $\widetilde{V}(f)$ the closure in $\mathbf{C}_{0}^{2}$ of $E^{-1}(V(f)-\{0\})$. Also, we will say that two varieties $V(f)$ and $V(g)$ coincide to order $k$ if their defining functions $f$ and $g$ coincide to order $k$ at 0 . 
Lemma 3.1. Let $f$ and $g$ be germs of irreducible holomorphic functions of order $j$ at $0 \in \mathbf{C}^{2}$. Suppose that $f$ and $g$ coincide to order $k(k>j)$ at 0 . Then $\widetilde{V}(f)$ and $\widetilde{V}(g)$ coincide to order $k-j$ on $E^{-1}(0)$.

Proof. Without loss of generality, we can assume that the tangent cone of $V(f)$ or $V(g)$ is not the point $(s=0, y=0)$. So the varieties $\widetilde{V}(f)$ and $\widetilde{V}(g)$ admit, respectively, $\tilde{f}(x, t)=f(x, t x) / x^{j}$ and $\tilde{g}(x, t)=g(x, t x) / x^{j}$ as defining functions. Therefore, it follows from the fact that $f$ and $g$ coincide to order $k$ at 0 , that $\tilde{f}(x, t)$ and $\tilde{g}(x, t)$ coincide to order $k-j$ on $E^{-1}(0)$.

Lemma 3.2. Suppose that $A, B$ are germs of holomorphic functions that coincide to order $k_{1}$ and $V(f), V(g)$ are germs of analytic varieties that coincide to order $k_{2}$ at $0 \in \mathbf{C}^{2}$. Then the images via the maps $F(x, y)=(x, A(x, y))$ and $G(x, y)=(x, B(x, y))$ of, respectively, $V(f)$ and $V(g)$ coincide to an order $\nu\left(k_{1}, k_{2}\right)$, where $\nu$ increases with $k_{1}, k_{2}$.

Proof. Let $p$ be the order of $f$ and $g$ at 0 . Then a parametrization of $V(f)$ (resp. $V(g))$ is (see [4])

$$
\left(x=t^{p}, y=a(t)\right) \quad\left(\text { resp. }\left(x=t^{p}, y=b(t)\right)\right),
$$

where $a, b$ are holomorphic functions of order $\geq p+1$. Moreover, it follows from the fact that $f$ and $g$ coincide to order $k_{2}$ that $a$ and $b$ coincide to an order $\eta\left(k_{2}\right)$, where $\eta$ increases with $k_{2}$. The parametrization of $F(V(f))$ (resp. $G(V(g)))$ is

$$
\left(x=t^{p}, y=A\left(t^{p}, a(t)\right)\right) \quad\left(\text { resp. }\left(x=t^{p}, y=B\left(t^{p}, b(t)\right)\right)\right) .
$$

The conclusion follows from the fact that $A, B$ coincide to order $k_{1}$ and $a$, $b$ coincide to order $\eta\left(k_{2}\right)$.

Proof of the theorem. We write

$$
M(x, y)=\frac{\alpha(x, y)}{\beta(x, y)}=\frac{\sum_{j \geq n} P_{j}(x, y)}{\sum_{j \geq n} Q_{j}(x, y)},
$$

where $P_{j}, Q_{j}$ are homogeneous polynomials of degree $j$ and $P_{n}, Q_{n}$ coprime. For $k>n$, define the rational function $R_{k}$ by

$$
R_{k}(x, y)=\frac{\alpha_{k}(x, y)}{\beta_{k}(x, y)}=\frac{\sum_{j=n}^{k} P_{j}(x, y)}{\sum_{j=n}^{k} Q_{j}(x, y)} .
$$

Note that the blow-up $\widetilde{M}$ of $M$ coincides on $E^{-1}(0)$ with the blow-up $\widetilde{R}_{k}$ of $R_{k}$, it is

$$
\widetilde{M}(0, t)=\widetilde{R}_{k}(0, t)=\frac{P_{n}(1, t)}{Q_{n}(1, t)} .
$$


Consider the maps $\mathscr{P}: \mathbf{C}_{0}^{2} \mapsto \mathbf{C}_{0}^{2}$ and $\mathscr{P}_{k}: \mathbf{C}_{0}^{2} \mapsto \mathbf{C}_{0}^{2}$, defined in the charts $(x, t),(s, y)$ by

$$
\begin{aligned}
& \mathscr{P}(x, t)=(x, \widetilde{M}(x, t)) \text { and } \mathscr{P}(s, y)=\left(\frac{1}{\widetilde{M}(s y, 1 / s)}, s y \widetilde{M}\left(s y, \frac{1}{s}\right)\right), \\
& \mathscr{P}_{k}(x, t)=\left(x, \widetilde{R}_{k}(x, t)\right) \text { and } \mathscr{P}_{k}(s, y)=\left(\frac{1}{\widetilde{R}_{k}(s y, 1 / s)}, s y \widetilde{R}_{k}\left(s y, \frac{1}{s}\right)\right) .
\end{aligned}
$$

Note that the meromorphic map $\mathscr{P}$ (resp. $\mathscr{P}_{k}$ ) is holomorphic outside the variety $\{\widetilde{M}=\infty\}$ (resp. $\left\{\widetilde{R}_{k}=\infty\right\}$ ). Since the jacobian of $\mathscr{P}$ (resp. $\mathscr{P}_{k}$ ), in the chart $(x, t)$, vanishes only on the variety

$$
X=\left\{(x, t) ; \frac{\partial \widetilde{M}}{\partial t}=0\right\} \quad\left(\text { resp. } X_{k}=\left\{(x, t) ; \frac{\partial \widetilde{R}_{k}}{\partial t}=0\right\}\right),
$$

then $\mathscr{P}$ (resp. $\mathscr{P}_{k}$ ) is a covering outside $X$ (resp. $X_{k}$ ) whose number of sheets is the multiplicity of $\widetilde{M}(0, t)$. Since

and

$$
E(X)=\left\{(x, y) ; \beta \frac{\partial \alpha}{\partial y}-\alpha \frac{\partial \beta}{\partial y}=0\right\}
$$

$$
E\left(X_{k}\right)=\left\{(x, y) ; \beta_{k} \frac{\partial \alpha_{k}}{\partial y}-\alpha_{k} \frac{\partial \beta_{k}}{\partial y}=0\right\}
$$

and the functions $\beta \frac{\partial \alpha}{\partial y}-\alpha \frac{\partial \beta}{\partial y}, \beta_{k} \frac{\partial \alpha_{k}}{\partial y}-\alpha_{k} \frac{\partial \beta_{k}}{\partial y}$ coincide to order $2 k-1$, then $X$ and $X_{k}$ coincide to an order $\eta(k)$ (see Lemma 3.1). Therefore the varieties $W=\mathscr{P}(X)$ and $W_{k}=\mathscr{P}_{k}\left(X_{k}\right)$ coincide to a certain order $\mu(k)$, and $\mu$ increases with $k$ (see Lemma 3.2).

Now, it follows from Proposition 2.1 that if $k$ is large enough, then there is a homeomorphism $\Psi:\left(\mathbf{C}^{2}, 0\right) \mapsto\left(\mathbf{C}^{2}, 0\right)$ such that

$$
\tilde{\Psi}(W)=W_{k} \quad \text { and } \quad \tilde{\Psi}\left(\left\{(x, t) ; t=t_{0}\right\}\right)=\left\{(x, t) ; t=t_{0}\right\},
$$

for every constant $t_{0}$. Next, since $\mathscr{P}$ (resp. $\mathscr{P}_{k}$ ) maps the analytic varieties $\widetilde{M}^{-1}\left(\widetilde{M}\left(x_{0}, t_{0}\right)\right)$ (resp. $\left.\widetilde{R}_{k}^{-1}\left(\widetilde{R}_{k}\left(x_{0}, t_{0}\right)\right)\right)$ into the complex line $t=t_{0}$, there is a homeomorphism $\widetilde{\Phi}$ such that the diagram

$$
\begin{aligned}
& \left(\mathbf{C}_{0}^{2}, X\right) \stackrel{\widetilde{\Phi}}{\longrightarrow}\left(\mathbf{C}_{0}^{2}, X_{k}\right) \\
& \left(\mathbf{C}_{0}^{2}, W\right) \stackrel{\widetilde{\Psi}}{\longrightarrow}\left(\mathbf{C}_{0}^{2}, W_{k}\right)
\end{aligned}
$$

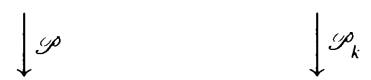

commutes, i.e., $\widetilde{\Phi}$ conjugates the foliation defined by the level sets of $\widetilde{M}$ to that defined by the level sets of $\widetilde{R}_{k}$. Since $\widetilde{M}=\widetilde{R}_{k}$ on $E^{-1}(0)$ and $\widetilde{\Psi}$ is the identity on $E^{-1}(0)$, then $\widetilde{\Phi}$ can be chosen to be the identity on $E^{-1}(0)$. Finally, the homeomorphism $\Phi=E \circ \widetilde{\Phi} \circ E^{-1}$, defined in a neighborhood of $0 \in \mathrm{C}^{2}$, conjugates $R_{k}$ with $M$, i.e., $R_{k} \circ \Phi=M$. The theorem is proved. 


\section{REFERENCES}

1. D. Cerveau and J. F. Mattei, Formes integrables holomorphes singulieres, Asterisque 97 (1982).

2. K. Kendig, Elementary algebraic geometry, Springer-Verlag, New York, 1977.

3. A. Meziani, On the integrability of singular differential forms in two complex variables, Ph.D. Thesis, Rutgers, 1988; Trans. Amer. Math. Soc. (to appear).

4. H. Whitney, Complex analytic varieties, Addison Wesley, Reading, MA, 1972.

Department of Mathematics, University Park, FIU, Miami, Florida 33199 